\title{
Charmed-Hadron Fragmentation Functions from CERN LEP1
}

\section{Revisited}

\author{
Bernd A. Knieh* and Gustav Kramel \\ II. Institut für Theoretische Physik, Universität Hamburg, \\ Luruper Chaussee 149, 22761 Hamburg, Germany
}

(Dated: February 13, 2007)

\begin{abstract}
In Phys. Rev. D 58, 014014 (1998) and 71, 094013 (2005), we determined non-perturbative $D^{0}$, $D^{+}, D^{*+}, D_{s}^{+}$, and $\Lambda_{c}^{+}$fragmentation functions, both at leading and next-to-leading order in the $\overline{\mathrm{MS}}$ factorization scheme, by fitting $e^{+} e^{-}$data taken by the OPAL Collaboration at CERN LEP1. The starting points for the evolution in the factorization scale $\mu$ were taken to be $\mu_{0}=2 m_{Q}$, where $Q=c, b$. For the reader's convenience, in this Addendum, we repeat this analysis for $\mu_{0}=m_{Q}$, where the flavor thresholds of modern sets of parton density functions are located.
\end{abstract}

PACS numbers: 13.60.-r, 13.85.Ni, 13.87.Fh, 14.40.Lb

*Electronic address: bernd.kniehl@desy.de

${ }^{\dagger}$ Electronic address: gustav.kramer@desy.de 


\section{INTRODUCTION}

The OPAL Collaboration presented measurements of the fractional energy spectra of inclusive $D^{*+}[1], D^{0}, D^{+}, D_{s}^{+}$, and $\Lambda_{c}^{+}[2]$ production in $Z$-boson decays based on their entire LEP1 data sample. Apart from the full cross sections, they also determined the contributions arising from $Z \rightarrow b \bar{b}$ decays. This enabled us, partly in collaboration with Binnewies, to determine lowest-order (LO) and next-to-leading-order (NLO) sets of fragmentation functions (FF's) for these charmed $\left(X_{c}\right)$ hadrons [3, 4]. We took the charm-quark FF to be of the form proposed by Peterson et al. [5] and thus obtained new values of the $\epsilon$ parameter, which are specific for our choice of factorization scheme.

We worked in the QCD-improved parton model implemented in the pure modified minimal-subtraction $(\overline{\mathrm{MS}})$ renormalization and factorization scheme with $n_{f}=5$ massless quark flavors (zero-mass variable-flavor-number scheme). This scheme is particularly appropriate if the characteristic energy scale of the considered production process, i.e., the center-of-mass energy $\sqrt{s}$ in the case of $e^{+} e^{-}$annihilation and the transverse momentum $p_{T}$ of the $X_{c}$ hadron in other scattering processes, is large compared to the bottom-quark mass $m_{b}$. Owing to the factorization theorem [6], the FF's defined in this scheme satisfy two desirable properties: (i) their scaling violations are ruled by the time-like Dokshitzer-GribovLipatov-Altarelli-Parisi (DGLAP) 7] evolution equations; and (ii) they are universal. Thus, this formalism is predictive and suitable for global data analyses.

We verified that the values of the branching and average momentum fractions of the various $c, b \rightarrow X_{c}$ transitions evaluated at LO and NLO using our FF's [3, 4] are in reasonable agreement with the corresponding results from OPAL [1, 2] and other experiments [8].

We tested the scaling violations of our $D^{0}, D^{+}, D_{s}^{+}$, and $\Lambda_{c}^{+}$FF's [4] by comparing the fractional energy spectra of these hadrons measured in non-resonant $e^{+} e^{-}$annihilation at $\sqrt{s}=10.55 \mathrm{GeV}[9], 29 \mathrm{GeV}$ [10], and 34.7 [11] with our LO and NLO predictions to find reasonable agreement. Since events of $X_{c}$-hadron production from $X_{b}$-hadron decay were excluded from the data samples at $\sqrt{s}=10.55 \mathrm{GeV}$, we obtained a clean test of our charm-quark FF's.

In Refs. 3, 4], the starting points $\mu_{0}$ for the DGLAP evolution in the factorization scale $\mu$ were taken to be $\mu_{0}=2 m_{Q}$, where $Q=c, b$. This choice is phenomenologically motivated by the observation that, in $e^{+} e^{-}$annhilation, which has been providing the most 
constraining input for the determinations of FF's, these values of $\mu_{0}$ represent the very production thresholds of the respective flavors. Unfortunately, this choice is inconsistent with the convention underlying modern sets of parton density functions (PDF's) [12], which prefer to place the flavor thresholds at $\mu_{0}=m_{Q}$. For the reader's convenience, in this Addendum to Refs. [3, 4], we thus repeat the analysis of that papers for the choice $\mu_{0}=m_{Q}$, so as to provide alternative LO and NLO sets of $X_{c}$ FF's that can be conveniently utilized together with those PDF's. The FF's presented below were already used as input for a NLO study 13. of charmed-meson hadroproduction in $p \bar{p}$ collisions, which yielded agreement within errors with data collected by the CDF Collaboration in run II at the Fermilab Tevatron 14]. We note in passing that, in the case of perturbatively induced FF's, which is quite different from the case of non-perturbative FF's (involving substantial intrinsic components) under consideration here, the choice $\mu_{0}=m_{Q}$ is more natural, since, at NLO, it avoids finite matching conditions at the flavor thresholds [15].

\section{RESULTS}

In the following, we concentrate on the most important results of Refs. 3, 4] that are affected by the shift in $\mu_{0}$. These include the fit parameters $N, \alpha, \beta$, and $\epsilon$ defining the $x$ distributions of the $Q \rightarrow X_{c}$ FF's $D_{Q}\left(x, \mu^{2}\right)$ at $\mu=\mu_{0}$,

$$
\begin{aligned}
& D_{c}\left(x, \mu_{0}^{2}\right)=N \frac{x(1-x)^{2}}{\left[(1-x)^{2}+\epsilon x\right]^{2}}, \\
& D_{b}\left(x, \mu_{0}^{2}\right)=N x^{\alpha}(1-x)^{\beta},
\end{aligned}
$$

the $\chi^{2}$ values per degree of freedom $\left(\chi^{2} /\right.$ d.o.f. $)$ achieved in the fits, and the branching fractions $B_{Q}(\mu)$ and average momentum fractions $\langle x\rangle_{Q}(\mu)$,

$$
\begin{aligned}
B_{Q}(\mu) & =\int_{x_{\mathrm{cut}}}^{1} d x D_{Q}\left(x, \mu^{2}\right), \\
\langle x\rangle_{Q}(\mu) & =\frac{1}{B_{Q}(\mu)} \int_{x_{\mathrm{cut}}}^{1} d x x D_{Q}\left(x, \mu^{2}\right),
\end{aligned}
$$

where $x_{\text {cut }}=0.1$, at $\mu=2 \mu_{0}$ and $M_{Z}$. In the present analysis, we adopt the up-to-date input information from our 2005 paper [4].

Our new results are presented in Tables [IV Comparing Tables 【II and IV with the corresponding tables in Refs. [3, 4], we observe that the branching and average momentum 
TABLE I: Fit parameters of the charm- and bottom-quark FF's in Eqs. (11) and (2), respectively, for the various $X_{c}$ hadrons at LO and NLO. The corresponding starting scales are $\mu_{0}=m_{c}=1.5 \mathrm{GeV}$ and $\mu_{0}=m_{b}=5 \mathrm{GeV}$, respectively. All other FF's are taken to be zero at $\mu_{0}=m_{c}$.

\begin{tabular}{|c|c|c|c|c|c|c|}
\hline$X_{c}$ & Order & & $N$ & $\alpha$ & $\beta$ & $\epsilon$ \\
\hline \multirow[t]{4}{*}{$D^{0}$} & LO & $c$ & 0.694 & $\cdots$ & $\ldots$ & 0.101 \\
\hline & & $b$ & 81.7 & 1.81 & 4.95 & 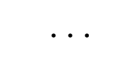 \\
\hline & NLO & $c$ & 0.781 & $\ldots$ & $\ldots$ & 0.119 \\
\hline & & $b$ & 100 & 1.85 & 5.48 & $\ldots$ \\
\hline \multirow[t]{4}{*}{$D^{+}$} & $\mathrm{LO}$ & $c$ & 0.282 & $\ldots$ & $\ldots$ & 0.104 \\
\hline & & $b$ & 52.0 & 2.33 & 5.10 & $\ldots$ \\
\hline & NLO & $c$ & 0.266 & $\ldots$ & $\ldots$ & 0.108 \\
\hline & & $b$ & 60.8 & 2.30 & 5.58 & $\ldots$ \\
\hline \multirow[t]{4}{*}{$D^{*+}$} & $\mathrm{LO}$ & $c$ & 0.174 & $\cdots$ & $\ldots$ & 0.0554 \\
\hline & & $b$ & 69.5 & 2.77 & 4.34 & $\cdots$ \\
\hline & NLO & $c$ & 0.192 & $\cdots$ & $\ldots$ & 0.0665 \\
\hline & & $b$ & 20.8 & 1.89 & 3.73 & $\ldots$ \\
\hline \multirow[t]{4}{*}{$D_{s}^{+}$} & LO & $c$ & 0.0498 & $\ldots$ & $\ldots$ & 0.0322 \\
\hline & & $b$ & 27.5 & 1.94 & 4.28 & $\ldots$ \\
\hline & NLO & $c$ & 0.0381 & $\ldots$ & $\ldots$ & 0.0269 \\
\hline & & $b$ & 27.5 & 1.88 & 4.48 & $\ldots$ \\
\hline \multirow[t]{4}{*}{$\Lambda_{c}^{+}$} & LO & $c$ & 0.00677 & $\ldots$ & $\ldots$ & 0.00418 \\
\hline & & $b$ & 41.2 & 2.02 & 5.92 & $\ldots$ \\
\hline & NLO & $c$ & 0.00783 & $\ldots$ & $\ldots$ & 0.00550 \\
\hline & & $b$ & 34.9 & 1.88 & 6.08 & $\ldots$ \\
\hline
\end{tabular}

fractions are changed very little by the reduction in $\mu_{0}$. For a comparison of these observables with experimental data, we refer to Refs. [3, 4].

For lack of space, we refrain from presenting here any updated versions of figures included in Refs. [3, 4]; they would not exhibit any qualitatively new features. However, as already mentioned in Ref. [13], the reduction in $\mu_{0}$ has an appreciable effect on the gluon FF's, which 
TABLE II: $\chi^{2} /$ d.o.f. achieved in the LO and NLO fits to the OPAL [1, 2] data on the various $X_{c}$ hadrons. In each case, $\chi^{2} /$ d.o.f. is calculated for the $Z \rightarrow b \bar{b}$ sample (b), the full sample (All), and the combination of both (Average).

\begin{tabular}{ccccc}
\hline \hline$X_{c}$ & Order & $b$ & All & Average \\
\hline$D^{0}$ & LO & 1.26 & 0.916 & 1.09 \\
& NLO & 1.10 & 0.766 & 0.936 \\
$D^{+}$ & LO & 0.861 & 0.658 & 0.759 \\
& NLO & 0.756 & 0.560 & 0.658 \\
$D^{*+}$ & LO & 1.19 & 1.12 & 1.16 \\
& NLO & 1.07 & 1.01 & 1.04 \\
$D_{s}^{+}$ & LO & 0.246 & 0.111 & 0.178 \\
& NLO & 0.290 & 0.112 & 0.201 \\
$\Lambda_{c}^{+}$ & LO & 1.05 & 0.117 & 0.583 \\
& NLO & 1.05 & 0.112 & 0.579 \\
\hline \hline
\end{tabular}

TABLE III: Branching fractions (in \%) of $Q \rightarrow X_{c}$ for $Q=c, b$ and the various $X_{c}$ hadrons evaluated according to Eq. (3) in LO and NLO at the respective production thresholds $\mu=2 m_{Q}$ and at the $Z$-boson resonance $\mu=M_{Z}$.

\begin{tabular}{cccccc}
\hline \hline$X_{c}$ & Order & $B_{c}\left(2 m_{c}\right)$ & $B_{c}\left(M_{Z}\right)$ & $B_{b}\left(2 m_{b}\right)$ & $B_{b}\left(M_{Z}\right)$ \\
\hline$D^{0}$ & LO & 72.8 & 67.6 & 57.5 & 52.7 \\
& NLO & 71.6 & 65.8 & 54.3 & 49.3 \\
$D^{+}$ & LO & 28.9 & 26.8 & 19.0 & 17.7 \\
& NLO & 26.4 & 24.3 & 18.5 & 17.1 \\
$D^{*+}$ & LO & 29.0 & 27.2 & 24.3 & 23.1 \\
& NLO & 27.8 & 25.9 & 24.5 & 22.8 \\
$D_{s}^{+}$ & LO & 12.3 & 11.7 & 23.1 & 21.2 \\
& NLO & 10.6 & 10.0 & 22.1 & 20.2 \\
$\Lambda_{c}^{+}$ & LO & 6.17 & 6.06 & 15.1 & 13.7 \\
& NLO & 6.12 & 5.87 & 14.3 & 12.8 \\
\hline \hline
\end{tabular}


TABLE IV: Average momentum fractions of $Q \rightarrow X_{c}$ for $Q=c, b$ and the various $X_{c}$ hadrons evaluated according to Eq. (4) in LO and NLO at the respective production thresholds $\mu=2 m_{Q}$ and at the $Z$-boson resonance $\mu=M_{Z}$.

\begin{tabular}{cccccc}
\hline \hline$X_{c}$ & Order $\langle x\rangle_{c}\left(2 m_{c}\right)$ & $\langle x\rangle_{c}\left(M_{Z}\right)$ & $\langle x\rangle_{b}\left(2 m_{b}\right)$ & $\langle x\rangle_{b}\left(M_{Z}\right)$ \\
\hline$D^{0}$ & LO & 0.573 & 0.442 & 0.318 & 0.285 \\
& NLO & 0.550 & 0.420 & 0.304 & 0.272 \\
$D^{+}$ & LO & 0.571 & 0.441 & 0.341 & 0.302 \\
& NLO & 0.557 & 0.425 & 0.324 & 0.287 \\
$D^{*+}$ & LO & 0.617 & 0.472 & 0.393 & 0.344 \\
& NLO & 0.592 & 0.448 & 0.366 & 0.322 \\
$D_{s}^{+}$ & LO & 0.654 & 0.496 & 0.348 & 0.310 \\
& NLO & 0.653 & 0.487 & 0.337 & 0.299 \\
$\Lambda_{c}^{+}$ & LO & 0.765 & 0.571 & 0.302 & 0.272 \\
& NLO & 0.738 & 0.544 & 0.290 & 0.261 \\
\hline \hline
\end{tabular}

are only feebly constrained by $e^{+} e^{-}$data. This effect is visualized for $X_{c}=D^{*+}$ in Fig. 1, where the $\mu_{0}=m_{Q}$ to $\mu_{0}=2 m_{Q}$ ratios of $D_{g}\left(x, \mu^{2}\right)$ at $\mu=5,10$, and $20 \mathrm{GeV}$ are shown as functions of $x$. We observe that the reduction in $\mu_{0}$ leads to a significant enhancement of the gluon FF, especially at low values of $x$. The results for $X_{c}=D^{0}, D^{+}, D_{s}^{+}$, and $\Lambda_{c}^{+}$are very similar and, therefore, not shown here.

\section{CONCLUSIONS}

In this Addendum to Refs. 3, 4], we repeated the fits of non-perturbative $D^{0}, D^{+}, D^{*+}$, $D_{s}^{+}$, and $\Lambda_{c}^{+} \mathrm{FF}$ 's, both at LO and NLO in the $\overline{\mathrm{MS}}$ factorization scheme, to OPAL data from LEP1 [1, 2] for the reduced choice $\mu_{0}=m_{Q}(Q=c, b)$ of starting point for the DGLAP evolution in the factorization scale $\mu$. These FF's are appropriate for use in connection with modern sets of PDF's [12], which are implemented with the same convention for the heavyflavor thresholds. A FORTRAN routine that evaluates the values of these FF's as functions of the input variables $x$ and $\mu$ may be obtained by electronic mail upon request from the authors. 
This reduction in $\mu_{0}$ is inconsequential for the theoretical interpretation of experimental $e^{+} e^{-}$data because it is compensated by corresponding shifts in the fit parameters $N, \alpha, \beta$, and $\epsilon$. However, the gluon FF's, which are only feebly constrained by $e^{+} e^{-}$data, play a significant role in hadroproduction. In fact, detailed analysis [13] revealed that the increase in the gluon FF's due to the extension of the evolution length leads to a rise in cross section and thus improves the agreement with the CDF data of charmed-meson production in run II at the Tevatron [14].

\section{ACKNOWLEDGMENTS}

We thank I. Schienbein and H. Spiesberger for useful discussions. This work was supported in part by the Bundesministerium für Bildung und Forschung through Grant No. 05 HT1GUA/4.

[1] OPAL Collaboration, K. Ackerstaff et al., Eur. Phys. J. C 1, 439 (1998).

[2] OPAL Collaboration, G. Alexander et al., Z. Phys. C 72, 1 (1996).

[3] J. Binnewies, B. A. Kniehl, and G. Kramer, Phys. Rev. D 58, 014014 (1998).

[4] B. A. Kniehl and G. Kramer, Phys. Rev. D 71, 094013 (2005).

[5] C. Peterson, D. Schlatter, I. Schmitt, and P. M. Zerwas, Phys. Rev. D 27, 105 (1983).

[6] J. C. Collins, Phys. Rev. D 58, 094002 (1998).

[7] V. N. Gribov and L. N. Lipatov, Yad. Fiz. 15, 781 (1972) [Sov. J. Nucl. Phys. 15, 438 (1972)]; G. Altarelli and G. Parisi, Nucl. Phys. B126, 298 (1977); Yu. L. Dokshitzer, Zh. Eksp. Teor. Fiz. 73, 1216 (1977) [Sov. Phys. JETP 46, 641 (1977)].

[8] L. Gladilin, Report No. hep-ex/9912064 (unpublished); DELPHI Collaboration, P. Abreu et al., Eur. Phys. J. C 12, 225 (2000); ALEPH Collaboration, R. Barate et al., ibid. 16, 597 (2000); S. Padhi, in Proceedings of the Ringberg Workshop on New Trends in HERA Physics 2003, edited by G. Grindhammer, B. A. Kniehl, G. Kramer, and W. Ochs, (World Scientific, Singapore, 2004), p. 183; H1 Collaboration, A. Aktas et al., Eur. Phys. J. C 38, 447 (2005).

[9] CLEO Collaboration, D. Bortoletto et al., Phys. Rev. D 37, 1719 (1988); CLEO Collaboration, R. A. Briere et al., ibid. 62, 072003 (2000); CLEO Collaboration, M. Artuso et al., ibid. 70, 112001 (2004). 
[10] HRS Collaboration, M. Derrick et al., Phys. Rev. Lett. 54, 2568 (1985); HRS Collaboration, P. Baringer et al., Phys. Lett. B 206, 551 (1988).

[11] TASSO Collaboration, M. Althoff et al., Phys. Lett. 136B, 130 (1984).

[12] A. D. Martin, R. G. Roberts, W. J. Stirling, and R. S. Thorne, Phys. Lett. B 604, 61 (2004); J. Pumplin, A. Belyaev, J. Huston, D. Stump, and W.-K. Tung, JHEP 0602, 032 (2006).

[13] B. A. Kniehl, G. Kramer, I. Schienbein, and H. Spiesberger, Phys. Rev. Lett. 96, 012001 (2006).

[14] CDF Collaboration, D. Acosta et al., Phys. Rev. Lett. 91, 241804 (2003).

[15] M. Cacciari, P. Nason, and C. Oleari, JHEP 0510, 034 (2005). 


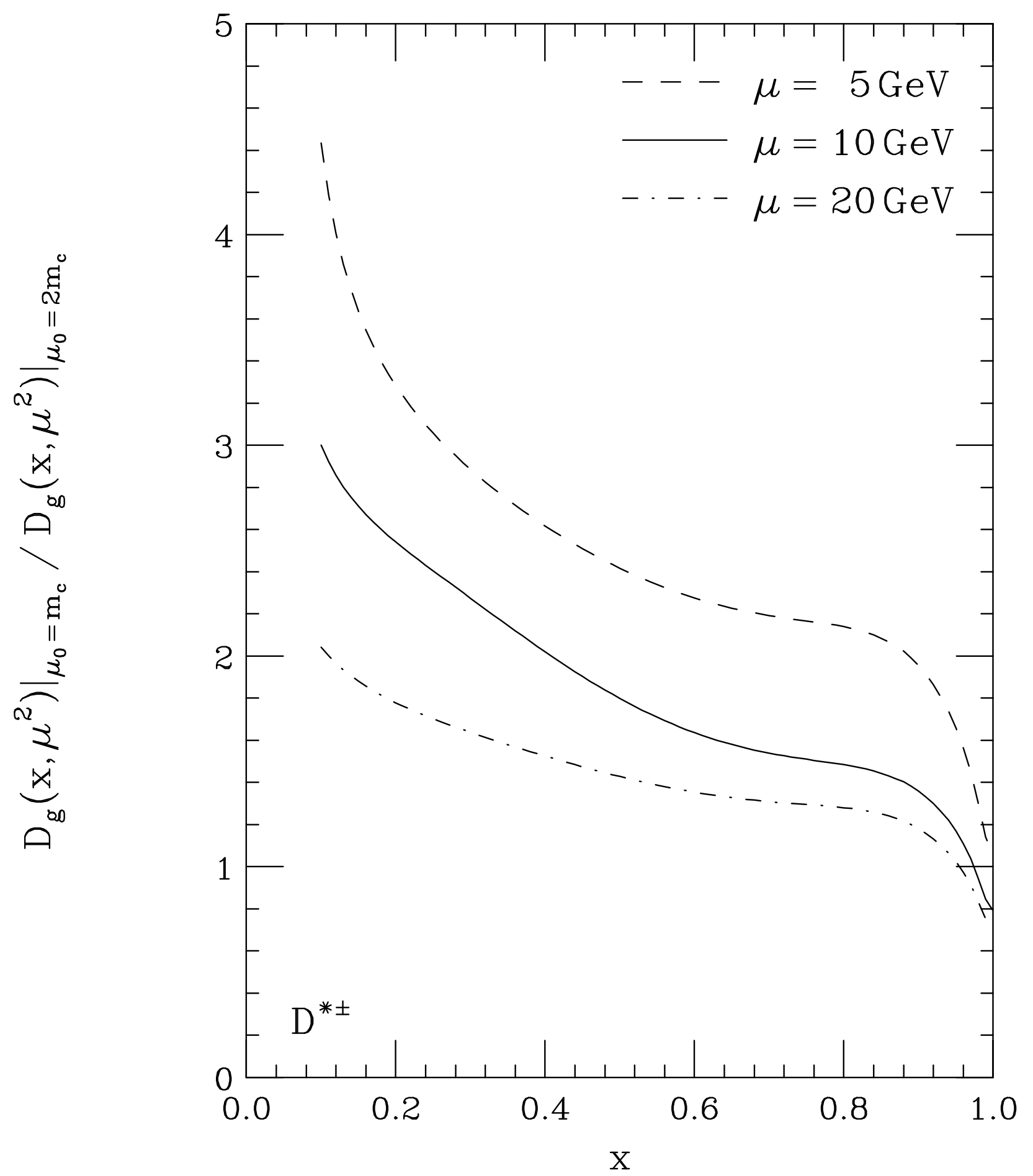

FIG. 1: $\mu_{0}=m_{Q}$ to $\mu_{0}=2 m_{Q}$ ratios of $D_{g}\left(x, \mu^{2}\right)$ at $\mu=5$ (dashed), 10 (solid), and $20 \mathrm{GeV}$ (dot-dashed) as functions of $x$ for $X_{c}=D^{*+}$. 